\title{
Religión y discapacidad: tramas que construyen la calidad de vida de los creyentes
}

\author{
Religion and disability: Weaves that build \\ the believers' quality of life
}

\author{
Carolina Mora Huertal y María Eugenia Patiño López ${ }^{2}$
} Citación: Mora H., C, y Patiño L., M.E. (2019). Religión y discapacidad: tramas que construyen la
calidad de vida de los creyentes. Psicología y Salud, 29(1), 125-137.

\section{RESUMEN}

\begin{abstract}
La calidad de vida es un aspecto de suma importancia para las personas que sufren discapacidades y para sus familias. La percepción, evaluación y aspiraciones que utilizan para valorar su vida están vinculadas a las creencias, algunas de ellas religiosas, ya que son las que suelen ofrecer respuestas al tránsito por el padecimiento o la muerte. El presente artículo recoge los principales hallazgos de un estudio cuyo objetivo fue conocer el papel que desempeñan las creencias religiosas en la construcción de la calidad de vida de familias con hijos con parálisis cerebral, desde el enfoque constructivista social y la metodología cualitativa. Se entrevistaron 53 familias, de las cuales 18 hablaron espontáneamente de sus creencias religiosas, sobre las cuales se centran la discusión y el análisis. Se observó que las creencias se convierten en la trama que conduce a estas familias a la abnegación por su adaptación a los valores culturales; al afrontamiento del problema o al cuestionamiento de esas creencias. Los datos recabados permiten establecer la importancia de las creencias religiosas en situaciones que implican cambios en la percepción y la estructura social de las familias.
\end{abstract}

Palabras clave: Calidad de vida; Creencias religiosas; Familia; Parálisis cerebral; Trama.

\begin{abstract}
Quality of life is an important issue for people with disabilities and their families. The perception, evaluation, and aspirations that people use to value their lives are tied to beliefs, some of them religious, since they offer some responses to illness and death. This aim of this study, which used a qualitative and socio-constructivist approach, was to identify the impact of religious beliefs in families with children with cerebral palsy. A total of 53 families were interviewed, 18 of which spoke spontaneously about their religious beliefs. Such beliefs seem to become a weave that lead participants to adopt abnegation by adapting to cultural values. These beliefs also affect coping, or even questioning such beliefs. The main findings allow to establish the importance of beliefs in situations involving perceptual and structural changes in families.
\end{abstract}

Key words: Quality of life; Religious beliefs; Family; Cerebral palsy; Weave.

\footnotetext{
${ }^{1}$ Centro de Rehabilitación e Inclusión Infantil Teletón Aguascalientes, Invierno 37, Fracc. Real Solare, 76246 El Marqués, Qro., México, tel. (449)198-04-92, correo electrónico: carolina_mh@yahoo.com.mx. Artículo recibido el 2 de abril y aceptado el 24 de mayo de 2018.

${ }^{2}$ Departamento de Sociología y Antropología, Centro de Ciencias Sociales y Humanidades, Universidad Autónoma de Aguascalientes, Av. Universidad 940, Ciudad Universitaria, 20131 Aguascalientes, Ags., México, tel. (449)910-84-90, correo electrónico: eugeniapatino@hotmail.com.
} 


\section{INTRODUCCIÓN}

$\mathrm{E}$ ntre los años 2010 y 2013 se realizó un estudio con 53 familias con hijos con diagnóstico de parálisis cerebral; en él se exploraron los atributos más importantes con los que ellas definían la calidad de vida, para lo cual se utilizaron como eje de la guía de entrevista las variables que la conforman, al tiempo que se exploraron algunos aspectos físicos, emocionales, cognitivos, sociales y culturales. A pesar de que la religión no fue uno de los aspectos que se consideraban en la entrevista, dieciocho de esas familias señalaron espontáneamente ese elemento como importante para su bienestar. Este grupo representó la tercera parte de los entrevistados. El presente trabajo da cuenta de la relación entre la religión y la calidad de vida encontrada en las entrevistas realizadas a este conjunto.

La familia es el grupo primario donde ocurre la socialización, y su organización suele obedecer a las características socioculturales del entorno. Cuando uno de sus miembros sufre un padecimiento como la parálisis cerebral infantil, la estructura experimenta cambios y adaptaciones en virtud de las interacciones que se desarrollan alrededor de la discapacidad. Tal reacomodo afecta a cada uno de los miembros de la familia y a ésta como grupo.

En la mayoría de los casos, es la madre quien cuida al niño, pero ello no la releva de sus otros roles dentro de la familia (madre, esposa, etc.). A ella no se le reconoce su derecho al bienestar, pues deben atender antes a sus hijos o pareja. El padre aparece como proveedor y casi siempre se mantiene periférico con respecto a las labores del hogar o el cuidado de los hijos, y los hermanos de los niños discapacitados frecuentemente asumen el papel de progenitores, o son hechos a un lado por el sistema familiar (Mora, 2012).

La percepción de la calidad de vida es algo de suma importancia para las personas discapacitadas y sus familias. La satisfacción en cuanto a sus condiciones de vida y salud define el modo en que afrontan las situaciones propias de su organización. Debido a sus circunstancias, buscan constantemente el bienestar individual y subjetivo, así como el social e intersubjetivo, esto es, su equilibro emocional y cognitivo, al tiempo que luchan por mantener sus interacciones sociales. Es aquí donde las creencias religiosas pueden desempeñar un importante papel al ser una fuente de estabilidad; en el caso de aquellos que asisten a las iglesias, pueden encontrar en algunos casos una red alterna de sociabilidad y ayuda.

El concepto calidad de vida ha sido definido desde muy diversos contextos y remite a una serie de aspectos es los que su empleo es diferente. Un recorrido por el estado de la cuestión remite a algunos de ellos. Schalock y Verdugo (2009), por ejemplo, entienden la calidad de vida como un concepto multidimensional que tiene los mismos componentes para todas las personas y que está influido por factores personales, ambientales y de interacción de carácter tanto objetivo como subjetivo. Las dimensiones a las que aluden dichos autores son, a saber: bienestar emocional, relaciones interpersonales, bienestar material, desarrollo personal, bienestar físico, autodeterminación, inclusión social y derechos.

Algunos estudios transculturales señalan la pertinencia de registrar las diferencias propias del entorno cuando se evalúa la calidad de vida. Verdugo, Jenaro y Schalock (2009) encontraron que en ciertas poblaciones latinoamericanas las dimensiones más significativas para las personas con discapacidad fueron el bienestar emocional, las relaciones interpersonales, el bienestar material, el bienestar físico y los derechos humanos y legales, mientras que para sus familiares fueron el bienestar emocional, el bienestar físico y los derechos humanos y legales.

En este trabajo se asume como "calidad de vida" la percepción, la evaluación y las aspiraciones relacionadas a las creencias de una persona o un grupo respecto a su experiencia vital en cuanto a su condición física, emocional, familiar y social, así como al bienestar que brinda el entorno en lo que corresponde al apoyo institucional en los ámbitos de la salud, recreación, oportunidades y convivencia, todo lo cual tiene su sustento en el contexto y la cultura en la que se desarrollan las personas.

De acuerdo con Salazar (2014), la explicación racional de las causas de una muerte sirve de poco y crea lo que Talcott Parsons (1996) define como un "problema de significado", un vacío semántico en la experiencia humana que la cultura tiende a llenar. Del instinto de preservación de la especie se deriva el miedo a la muerte y a las en- 
fermedades, y también la necesidad de alimentarse, pero no se satisface únicamente con comportamientos instintivos: los humanos necesitan de la cultura, una parte fundamental de la cual se conforma a través de las creencias, las que, según Caballero (2014), son ideas que ayudan al individuo a responder en el mundo. En este sentido, las creencias religiosas influyen considerablemente en la manera en que las personas se conducen en situaciones diversas y cotidianas, desde las más simples hasta las más demandantes, como la enfermedad propia o la de algún otro miembro de la familia.

Pese a ello, las creencias religiosas pueden actuar de distintas maneras en el afrontamiento de la enfermedad. En estudios como el llevado a cabo por Yanez et al. (2009) en una muestra de 418 pacientes diagnosticados con cáncer, se encontró que el propósito y la paz se relacionaban con una menor depresión, mientras que la religiosidad se vinculó con su aumento. En la investigación de Ridge, Williams, Anderson y Elford (2008) sobre las creencias religiosas como un medio de afrontamiento para personas con VIH/sida, se halló que la religión ayudaba a tener un sentimiento de pertenencia y brindaba seguridad por la claridad de sus reglas. A pesar del estigma que las instituciones religiosas instigan respecto a los padecimientos como el VIH, al que se asocia con la promiscuidad y la drogadicción, los participantes descubren que su fe les proporciona ayuda como soporte y esperanza. Se encuentra que la religión también provee paz mental, evita que se acentúe la condición sufriente y promueve pensamientos positivos. Para Funes (2007), a pesar de que la religión se asocia con la estigmatización de ciertos padecimientos, es una fuente de fuerza, apoyo, coraje y esperanza. Se observa en estos estudios que la pesquisa sobre las creencias y prácticas religiosas de las personas con un padecimiento puede generar un conocimiento que sirva para el diseño de estrategias terapéuticas que fomenten el bienestar de los sujetos involucrados, y el presente trabajo no es una excepción.

Como se puede advertir, a pesar de que algunas investigaciones sugieren que las creencias religiosas contribuyen a la percepción de una mejor calidad de vida, ciertos hallazgos muestran que en realidad no favorecen el bienestar y hacen que el entendimiento del mundo sea más "aterrador" (Boyer, 2010, p. 38). Bateson y Bateson (2000) señalan que las creencias religiosas suelen ser un paliativo, pero a la vez advierten acerca del error que entraña emplearlas como un anestésico para el sufrimiento crónico. Ello no implica que no se deba indagar sobre las creencias y prácticas de las personas enfermas y de sus familias; por el contrario, esa tarea es una guía para llevar a los sujetos a una atención integral.

Ambas perspectivas -las creencias religiosas como estrategias de afrontamiento o causantes de angustia- son posibles cuando se habla de una enfermedad discapacitante, grave o degenerativa. Según Kleinman (1988), la naturaleza de las narrativas y las características de las tramas con las que se explican las situaciones vitales -entre ellas las creencias-suelen determinar los modos de entender y hacer frente a la enfermedad y el sufrimiento. Esto traza un camino en el que es posible advertir que para algunos grupos (tales como las familias con un miembro con discapacidad) las creencias religiosas desempeñan un papel significativo en cuanto al modo de construir su realidad ante su medio social.

En el presente trabajo se buscó estudiar aquellos elementos que esos grupos toman de sus creencias religiosas para comprender la manera en que conciben su experiencia y, por lo tanto, perciben el modo en que viven, lo que parece importante en virtud de que traen consigo el bienestar o atormentan a los que sufren, pues esto los llevará a responder a su situación de un modo correspondiente e influirá en su experiencia de la enfermedad. Tal conocimiento puede ser útil a los profesionales de las diferentes disciplinas que atienden cuestiones relativas a la salud (antropología, enfermería, medicina, psicología, terapia física y ocupacional, trabajo social, etc.) y a los organismos de la sociedad encargados de las políticas públicas en salud, con el fin de favorecer a las personas enfermas o discapacitadas y a sus familias.

\section{MÉTODO}

El diseño metodológico fue cualitativo y parte de la propuesta de los métodos narrativos, utilizando como técnica de obtención de información la en- 
trevista a profundidad, para posteriormente aplicar un análisis de contenido según la propuesta de Adréu (2000).

\section{Muestra}

El grupo de muestra estuvo conformado por familias inscritas en el Centro de Rehabilitación e Inclusión Infantil Teletón Aguascalientes (CRIT), uno de cuyos miembros sufriera parálisis cerebral infantil, las cuales se seleccionaron por muestreo teórico, lo que supone "una representación no estadística, sino la representación tipológica socioestructural correspondiente a los objetivos del estudio" (Vallés, 2009, p. 68). Así, se entrevistó a 53 familias, de las cuales, para efectos del presente artículo, se retomaron 18 por ser las que relacionaron espontáneamente sus creencias religiosas con los indicadores de calidad de vida. En todos los casos se respetó el anonimato, por lo que en los resultados se utilizan seudónimos para una exposición más clara. La guía de entrevista fue construida a partir de ocho preguntas-eje de las que se derivaron otras adicionales de acuerdo con la situación particular.

\section{Procedimiento}

Se convocó a la familia completa, aunque la mayor parte de los casos fueron las madres las que acudían a las entrevistas con sus hijos discapacitados. En un caso asistió el padre, en otro la abuela, y únicamente en dos casos asistieron el padre, la madre y uno de los hermanos. Generalmente este hecho se pudo atribuir a los horarios de trabajo, pues mientras los padres iban a laborar, las madres quedaban al cuidado de los menores.

Las entrevistas se hicieron en dos sesiones con duración de 40 minutos en promedio.

\section{RESULTADOS}

Las características de las familias se muestran en las Tablas 1, 2 y 3 según la severidad del padecimiento.

Tabla 1. Características de las familias de los niños con parálisis cerebral severa.

\begin{tabular}{|c|c|c|c|c|c|c|}
\hline Caso & Estrato & $\begin{array}{c}\text { Tipo } \\
\text { de familia }\end{array}$ & Miembros & Edad & Escolaridad & Ocupación \\
\hline \multirow{5}{*}{1} & \multirow{5}{*}{ Alto } & \multirow{5}{*}{ Nuclear } & Padre & 44 & Primaria & Gobierno \\
\hline & & & Madre & 45 & Secundaria & Comercio \\
\hline & & & Hija & 20 & Licenciatura & \\
\hline & & & Hijo & 16 & High School & \\
\hline & & & Hija (paciente) & 12 & Primaria & \\
\hline \multirow{5}{*}{2} & \multirow{5}{*}{ Medio } & \multirow{5}{*}{ Nuclear } & Padre & 36 & Primaria no terminada & Mecánico \\
\hline & & & Madre & 35 & Primaria & Comercio \\
\hline & & & Hija & 16 & Secundaria & \\
\hline & & & Hijo & 8 & Primaria & \\
\hline & & & Hijo (paciente) & 3 & Escuela especial & \\
\hline \multirow{8}{*}{3} & \multirow{8}{*}{ Medio } & \multirow{8}{*}{ Nuclear } & Padre & 47 & Primaria trunca & Comerciante \\
\hline & & & Madre & 43 & Primaria & Hogar \\
\hline & & & Hijo & 26 & Preparatoria & Trabaja en EU \\
\hline & & & Hijo & 24 & Secundaria & Albañil \\
\hline & & & Hija & 22 & Preparatoria & Hogar \\
\hline & & & Hija & 20 & Preparatoria & Tendera \\
\hline & & & Hija & 11 & Primaria & \\
\hline & & & Hijo (paciente) & 5 & Escuela especial & \\
\hline
\end{tabular}




\begin{tabular}{|c|c|c|c|c|c|c|}
\hline \multirow{4}{*}{4} & \multirow{4}{*}{ Medio } & \multirow{4}{*}{$\begin{array}{l}\text { Monoparental / } \\
\text { madre presente }\end{array}$} & Padre & 27 & Licenciatura & \\
\hline & & & Madre & 31 & Licenciatura & Hogar \\
\hline & & & Hijo & 8 & Primaria & \\
\hline & & & Hija (paciente) & 7 & Escuela especial & \\
\hline \multirow{7}{*}{5} & \multirow{7}{*}{ Bajo } & \multirow{7}{*}{ Nuclear } & Padre & 44 & Primaria & Plomero \\
\hline & & & Madre & 36 & Primaria trunca & Hogar \\
\hline & & & Hijo & 17 & Preparatoria & \\
\hline & & & Hijo & 16 & Analfabeta & \\
\hline & & & Hija & 10 & Primaria & \\
\hline & & & Hija (paciente) & 5 & Escuela especial & \\
\hline & & & Hijo & $2 \mathrm{~m}$ & & \\
\hline \multirow{7}{*}{6} & \multirow{7}{*}{ Bajo } & \multirow{7}{*}{ Nuclear } & Padre & 47 & Secundaria & Mecánico \\
\hline & & & Madre & 49 & Licenciatura & Tendera, hogar \\
\hline & & & Hija & 23 & & Comercio \\
\hline & & & Hijo & 22 & & \\
\hline & & & Hija & 20 & Preparatoria & \\
\hline & & & Hija (paciente) & 15 & Escuela especial & \\
\hline & & & Hijo & 12 & Primaria & \\
\hline \multirow{4}{*}{7} & \multirow{4}{*}{ Medio } & \multirow{4}{*}{ Nuclear } & Padre & 39 & Analfabeta & Jornalero \\
\hline & & & Madre & 33 & Analfabeta & Hogar \\
\hline & & & Hija & 16 & Secundaria & \\
\hline & & & Hija (paciente) & 9 & Sin estudios & \\
\hline \multirow{5}{*}{8} & \multirow{5}{*}{ Medio } & \multirow{5}{*}{ Nuclear } & Padre & 38 & Preparatoria & Empleado \\
\hline & & & Madre & 37 & Primaria & Hogar \\
\hline & & & Hijo (paciente) & 13 & Sin estudios & \\
\hline & & & Hija & 12 & Secundaria & \\
\hline & & & Hijo & 10 & Primaria & \\
\hline \multirow{11}{*}{9} & \multirow{11}{*}{ Medio } & \multirow{11}{*}{$\begin{array}{l}\text { Monoparental / } \\
\text { madre presente }\end{array}$} & Padre & 45 & Secundaria & Preso \\
\hline & & & Madre & 42 & Analfabeta & Diversos trabajos \\
\hline & & & Hijo & 28 & Primaria & Albañil \\
\hline & & & Hijo & 26 & Analfabeta & Albañil \\
\hline & & & Hija & $8 d$ & $\begin{array}{l}\text { Finada insuficiencia } \\
\text { respiratoria }\end{array}$ & \\
\hline & & & Hija & 22 & Secundaria & Hogar \\
\hline & & & Hijo & 20 & Analfabeta & Albañil \\
\hline & & & Hijo & 18 & Primaria & Obrero \\
\hline & & & Hijo & 17 & Primaria & Mandados \\
\hline & & & Hijo & 15 & Primaria & Hace crema \\
\hline & & & Hija (paciente) & 14 & Escuela especial & \\
\hline \multirow{5}{*}{10} & \multirow{5}{*}{ Medio } & \multirow{5}{*}{ Nuclear } & Padre & 38 & Secundaria & Repartidor \\
\hline & & & Madre & 33 & Secundaria & Hogar \\
\hline & & & Hija & 15 & Secundaria & \\
\hline & & & Hijo & 14 & Secundaria & \\
\hline & & & Hijo (paciente) & 10 & & \\
\hline \multirow{4}{*}{11} & & & Padre & 28 & Primaria & Comercian \\
\hline & Bain & Nulear & Madre & 27 & Primaria & Hogar \\
\hline & Вајо & Nuciear & Hija & 9 & Primaria & \\
\hline & & & Hijo (paciente) & 6 & & \\
\hline
\end{tabular}


Tabla 2. Características de las familias de los niños con parálisis cerebral moderada.

\begin{tabular}{|c|c|c|c|c|c|c|}
\hline Caso & Estrato & $\begin{array}{c}\text { Tipo } \\
\text { de familia }\end{array}$ & Miembros & Edad & Escolaridad & Ocupación \\
\hline \multirow{9}{*}{12} & \multirow{9}{*}{ Medio } & \multirow{9}{*}{ Nuclear } & Padre & 50 & Secundaria & Plomero \\
\hline & & & Madre & 49 & Primaria & Hogar \\
\hline & & & Hijo (paciente) & 32 & preparatoria & Plomero \\
\hline & & & Hijo & 28 & Preparatoria & Plomero \\
\hline & & & Hija & 27 & Preparatoria & Hogar \\
\hline & & & Hijo & 19 & prepa & Empleado \\
\hline & & & Hijo & 18 & Licenciatura & \\
\hline & & & Hija & 16 & Preparatoria & \\
\hline & & & Hijo (paciente) & 12 & Escuela especial & \\
\hline \multirow{6}{*}{13} & \multirow{6}{*}{ Bajo } & \multirow{6}{*}{ Nuclear } & Padre & 31 & Primaria no terminada & Camionero \\
\hline & & & Madre & 32 & Analfabeta & Hogar \\
\hline & & & Hija & 14 & Primaria & \\
\hline & & & Hija & 11 & Primaria & \\
\hline & & & Hijo & 10 & Primaria & \\
\hline & & & Hijo (paciente) & 6 & Primaria & \\
\hline \multirow{4}{*}{14} & \multirow{4}{*}{ Bajo } & \multirow{4}{*}{ Nuclear } & Padre & 30 & Primaria & Jornalero \\
\hline & & & Madre & 31 & Primaria & Hogar \\
\hline & & & Hija & 9 & Primaria & Mostrador \\
\hline & & & Hijo (paciente) & 5 & & \\
\hline \multirow{4}{*}{15} & \multirow{4}{*}{ Bajo } & \multirow{4}{*}{ Nuclear } & Padre & 37 & Sin estudios & Albañil \\
\hline & & & Madre & 31 & Sin estudios & Hogar \\
\hline & & & Hijo & 10 & Primaria & \\
\hline & & & Hija (paciente) & 9 & Primaria & \\
\hline
\end{tabular}

Tabla 3. Características de las familias de los niños con parálisis cerebral leve.

\begin{tabular}{|c|c|c|c|c|c|c|}
\hline Caso & Estrato & $\begin{array}{c}\text { Tipo } \\
\text { de familia }\end{array}$ & Miembros & Edad & Escolaridad & Ocupación \\
\hline \multirow{4}{*}{16} & \multirow{4}{*}{ Medio } & \multirow{4}{*}{$\begin{array}{l}\text { Monoparental/ } \\
\text { madre presente }\end{array}$} & Padre & 42 & $\begin{array}{l}\text { Fallecido } \\
\text { por accidente }\end{array}$ & \\
\hline & & & Madre & 41 & & Hogar \\
\hline & & & Hijo & 12 & Primaria & \\
\hline & & & Hijo & 5 & Kínder & \\
\hline \multirow{5}{*}{17} & \multirow{5}{*}{ Bajo } & \multirow{5}{*}{ Nuclear } & Padre & 36 & Sin estudios & Desempleado \\
\hline & & & Madre & 34 & Sin estudios & Hogar \\
\hline & & & Hija & 9 & Primaria & \\
\hline & & & Hijo & 7 & Primaria & Trabaja en EU \\
\hline & & & Hijo (paciente) & 2 & & \\
\hline \multirow{7}{*}{18} & \multirow{7}{*}{ Medio } & \multirow{7}{*}{ Tres generaciones } & Abuelo & 62 & Secundaria & Pensionado \\
\hline & & & Abuela & 60 & Primaria no terminada & Hogar \\
\hline & & & Padre & 38 & Secundaria & Mostrador \\
\hline & & & Madre & 34 & Analfabeta & Prostituta \\
\hline & & & Hijo & 12 & Primaria & \\
\hline & & & Hijo (paciente) & 10 & Primaria & \\
\hline & & & Hija & 9 & Primaria & \\
\hline
\end{tabular}


En cuanto a su lugar de residencia, seis de esas familias residían en el estado de Zacatecas, cinco en Aguascalientes, cinco en Jalisco, una en San Luís Potosí y una en la ciudad de Houston, Texas. Los padres tenían en promedio una edad de treinta y nueve años, la mayoría con educación primaria. Las madres, un promedio de treinta y cuatro años, la mayor parte sin educación formal. Entre ellas hubo el mayor número de analfabetas (cuatro), o bien sin estudios, aunque sabían leer y escribir (cuatro).

Los niños con discapacidad tenían un diagnóstico de parálisis cerebral, nueve de ellos con discapacidad severa, cuatro con discapacidad moderada y tres con discapacidad leve ${ }^{3}$.

Respecto a su edad y lugar en la familia, los niños mencionados tenían un promedio de ocho años, y trece de ellos ocupaban el lugar de hijo menor. Seis asistían a una escuela regular, seis a una especial y seis no estaban escolarizados. Doce eran varones y seis mujeres.

\section{Características familiares}

Las familias entrevistadas sufrían diversos problemas asociados a la discapacidad. En primer lugar, había un quebranto en el vínculo entre los padres, ya sea por el sentimiento de culpa sobre el padecimiento del hijo -mismo que suele acompañar a muchos de estos casos- o por la dinámica de la división social del trabajo, en la que uno de ellos permanecía junto al hijo discapacitado y el otro se hacía cargo del sustento; es decir, uno era cuidador y el otro proveedor. En segundo término, dicha situación puede verse acompañada por el aislamiento de toda la familia de otros grupos sociales, lo que se acentúa en el cuidador primario, que en la mayoría de las familias es la madre. Algunas de estas mujeres mostraban depresión o ideación suicida, pero encontraban poco viable rendirse debido a que los menores necesitaban sus cuidados. Un tercer elemento fue la posición de

\footnotetext{
${ }^{3}$ Una clasificación de la parálisis cerebral se basa en su severidad. Una parálisis cerebral leve se caracteriza por la presencia de una alteración, pero no impide el desarrollo de las actividades en la vida cotidiana; moderada refiere a que la persona necesita de apoyos o asistencia para algunas acciones, y la severa implica la necesidad de apoyo para todas las actividades diarias (Lorente et al., 1993, p. 30).
}

los hermanos, algunos de ellos ignorados o dejados en los hogares de otros familiares por la imposibilidad de cuidarlos, y otros exhibían conductas asociales y violentas, o se adaptaban a la situación familiar a través de la realización de labores domésticas o el cuidado de los padres o del hermano discapacitado. En la mayoría de los casos, esta última alternativa parecía ser la más conveniente para la dinámica familiar.

\section{Presencia de la divinidad en los relatos}

Fue frecuente el uso de frases como "Dios aprieta, pero no ahorca" y "A veces amanecemos sin nada, pero Dios siempre se acuerda de uno". Aparece también la idea de un dios que ayuda a quitar el dolor, a cumplir deseos o a que los niños se integren a la escuela. Dios cuida, Dios ayuda a obtener trabajo y dinero, Dios es alguien con quien pueden hablar sobre la posible muerte de su hijo; Dios permite la salud; Dios es un ser que "quiere" que pasen cosas malas a los niños. Cuando se hizo un acercamiento a cada una de las narrativas, se hallaron historias con diferentes matices y significados. A continuación se hace una exposición en presente de los casos más relevantes para el análisis propuesto.

\section{Narrativas de los cuidadores principales}

\section{Caso 1. "No olvidarnos de nuestra religión".}

La familia de Luis (nueve años) está conformada por sus padres: Raúl (37 años, sin estudios) y Rosa (31 años, sin estudios); él y su hermano Vicente (10 años) asisten a la primaria. El padre se encuentra desvinculado emocionalmente de la familia y separado de ellos, sobre todo de la madre, de modo que no la ayuda en las labores del hogar ni en la rehabilitación de Luis, pues pasa la mayor parte de su tiempo con sus propios padres y hermanos, lo que hace que Rosa se sienta sola y desplazada.

Vicente y Luis juegan juntos y llevan una buena relación entre sí. Rosa se preocupa mucho por ellos y por Raúl, porque fuma mucho. La percepción que tiene de sí misma es que no piensa mucho en ella. Se descuida, cree que es egoísta dedicar más tiempo a ella que a sus hijos. Afirma que no puede pensar en otra cosa que no sean 
ellos. A pesar de esto, en ocasiones debe desempeñar tantas actividades en casa como cuidadora primaria, madre y esposa que no se ocupa de que Luis lleve a cabo su terapia de manera adecuada.

La familia desatiende en general los aspectos físicos de su calidad de vida. En cuestiones emocionales, Raúl y Rosa no se apoyan recíprocamente; para los hijos, la madre es su soporte. El aislamiento es una de las características de toda la familia en general, pues ninguno de sus miembros tiene amigos, y la socialización hacia afuera consiste en encuentros ocasionales con los vecinos o, en el caso de los niños, con los compañeros de la escuela. Cuando se les pregunta qué es calidad de vida para ellos, Rosa responde:

"Estar bien con la familia. Como nosotros, que vivimos con mis papás; llevarnos bien con ellos, con mis hermanos que están allí, saber convivir; o sea, estar bien. Estar bien ahora, sí, por estar en la gracia de Dios, vivir bien con uno mismo, y también no olvidarnos de nuestra religión. Recibir los sacramentos. Que ellos vayan a la escuela [señala a su hijo]. Estar bien alimentados. Casi significa lo mismo que la salud: si no tenemos qué comer, ¿cómo vamos a tener buena salud?”.

A pesar de que estar bien con Dios sea uno de los elementos principales para el bienestar, este no es suficiente para considerar satisfactoria su vida. A Rosa y a Luis les gustaría que cambiara y tener más convivencia con otras personas. Para Luís sería deseable poder caminar para sentirse satisfecho.

Caso 2. "Pídele a Dios que sea el diagnóstico de otro niño, de otra persona".

La familia está integrada por Guillermina (37 años, con estudios de primaria), Jorge (38 años, con preparatoria) y sus tres hijos: Rogelio (13 años, en escuela especial), Violeta (12 años, en secundaria) y Leonardo (10 años, en primaria). En esta familia prevalece la creencia de tipo providencialista en la que Dios es quien decide lo que ocurre, ya sea bueno o malo. Entre esos sucesos está la salud. Es Dios quien resuelve si los niños tendrán discapacidad o no, o cuánto tiempo vivirán. Con base en estas creencias, construyen la idea de que la discapacidad es el resultado de la voluntad divina.
«No, no era maligno [el tumor de una de las hijas], era un trauma muy raro. Pero yo por eso le digo que hemos estado unidos mi esposo y yo. Y mucha gente. Decía la mama de mi esposo: "No hijo, pídele a Dios que sea el diagnóstico de otro niño, de otra persona". "Pero si Dios quiso mandárnoslo a nosotros -decía mi esposo-, yo no quiero que nadie pase por esto que yo estoy pasando, por lo que nosotros estamos pasando. Si Dios así lo decidió, a echarle ganas y para adelante"».

La familia en general piensa que es una disposición de Dios la salud de los hijos, a tal grado que dejaron de llevar al niño a rehabilitación, ya que desde su perspectiva sólo necesitan de la fe para que el niño se encuentre bien. La confianza en Dios y sus decisiones es absoluta, por lo que la creencia religiosa es de suma importancia como un referente para comprender el mundo.

\section{Caso 3. "Ya Dios, si te lo quieres llevar, llévatelo".} Las creencias de los padres de familia cumplen un papel muy importante en los momentos críticos, como la muerte de los niños. Es el caso de Catalina (27 años, primaria), quien meses antes del fallecimiento de su hijo Antonio (de seis años) tuvo continuos momentos de negociación con la voluntad de Dios. A pesar de tener la convicción de que Dios era quien decidía si su hijo moría o no, sufría largos periodos de tristeza e incluso ideas sobre suicidio por su incapacidad para encontrar consuelo. La idea de la muerte de su hijo fue un problema difícil de sobrellevar:

"“iYa Dios, si te lo quieres llevar, llévatelo!" [...] Entonces, yo decía esas palabras en el hospital, pero cuando llegué a mi casa, al ver los recuerdos de Antonio, al ver sus juguetes en el ropero, su cuna donde él dormía, o sea, ¡no! Decía: "No, ¿cómo que te me vas a ir? Todavía no estoy preparada para que te vayas". Y es lo que quiero: sentirme preparada para el día de mañana poder decir: "Mi niño descansa en paz", y no decir: “¿Por qué te me vas?”»"

Las dificultades de su vida cotidiana no ayudan en este proceso, pues se trata de una familia en una situación funcionalmente difícil. En general, sus miembros se encuentran aislados, sin redes de apoyo de las familias extensas, sobre todo la de la madre. Catalina y su esposo Nuno (28 años, primaria) tienen una relación muy conflictiva y se 
encuentran desvinculados emocionalmente. Catalina se refugia en sus creencias religiosas para sobrellevar la situación en la que se encuentra.

Caso 4. "Si Dios nos dio otra oportunidad, ¿para qué nos vamos a estar lamentando?”.

En la familia de Sara (32 años, analfabeta) y Julián (31 años, sin estudios), el niño adquiere la parálisis cerebral debido a un accidente automovilístico en el que el padre pierde el control del vehículo. Ese día, Julián había bebido demasiado alcohol. Cecilio (seis años, primaria) viajaba en el asiento del copiloto en brazos de Sara. Cuando la camioneta choca, el niño es expulsado del vehículo y sufre daño cerebral. A partir de ese día, Julián culpa a Sara de lo ocurrido, y ella a su vez lo culpa a él.

La convivencia en familia es muy difícil debido a la violencia que Julián ejerce sobre Sara y sus cuatro hijos: Martín (14 años, primaria), Ania (11 años, primaria), Guillermo (10 años, primaria) y el propio Cecilio. Lo que más molesta a Sara es que Julián ponga a sus hijos a hacer ladrillos, que no los deje jugar y que desquite su enojo con ellos. Esta situación y el aislamiento tienen a Sara muy desilusionada. Aun así, ella ha hecho todo lo posible por mantener unida a la familia, y la manera en que lo ha logrado es resignificando el accidente en el que Cecilio sufrió el daño cerebral:

«No, a él [a Cecilio] nunca lo he visto así [que esté discapacitado por mi culpa]. Yo en un principio decía que era por nuestra culpa -de los dos, tanto él como yo-, pero digo: "Si Dios nos dio otra oportunidad, ¿para qué nos vamos a estar lamentando? En vez de estarnos lamentando, hay que darle lo mejor para que salga adelante porque ya Dios bendito nos dio licencia [para] que el niño esté otra vez con nosotros"»».

De esta forma, a pesar de la tristeza que padece, recupera la experiencia como una nueva oportunidad de vivir dada por Dios a pesar de las secuelas del accidente sufridas por el menor de sus hijos.

Caso 5. "Dios nos da lo que merecemos. A lo mejor es para mí salvación”.

En muchas de las representaciones que se tienen sobre la discapacidad, se asocia el hijo que la su- fre con la idea de que es la cruz que debe llevarse, tal como Jesucristo cargó la suya. Así, el menor se convierte en el sacrificio que debe hacer cada persona de manera diferente. Este es el caso de la familia de Dulce (13 años, en escuela de educación especial), integrada por sus padres Mauro (46 años, secundaria), Luz (42 años, analfabeta) y siete hermanos, de los cuales sólo dos viven actualmente con Luz y Dulce: León (17 años, primaria) y Ramón (15 años, primaria).

Luz se ha dedicado a cuidar y mantener a sus hijos sola, en un entorno sociocultural muy poco propicio para las mujeres ya que su esposo se encuentra preso. Su situación es de gran vulnerabilidad al no contar con trabajo ni tener la colaboración de su esposo. El medio machista en el que viven se perpetúa en los discursos cotidianos, incluso en los de Luz: "Una mujer, cuando quiere a su esposo, lo obedece, le tiene su ropa limpia, le tiene su cuarto limpio, le tiene su familia, lo atiende bien, con amor".

Actualmente ella está deprimida y con muchas carencias ya que no puede conseguir un trabajo con sueldo fijo. Para ella, sus hijos y la situación de su esposo son la cruz que debe llevar:

«Casi no estoy enojada; más bien me da por estar triste. Pero así de que maldiga o no maldiga, no... y menos a mis hijos. Rara es la vez que ando enojada. Yo siempre tengo una sonrisa en la cara, aunque ande que me lleva... La gente me dice: "Ay, señora, usted siempre bien contenta; usted siempre bien alegre y no aparenta la vida que lleva". Digo, es que la gente no tiene la culpa de mis problemas. Dicen mis hijos que cada quien se traza su destino. ¿Por qué voy a envidiar a otras personas? Dios nos da lo que merecemos, y a lo mejor es para mí salvación [señala a la niña con parálisis cerebral]. No sé. Me da por llorar».

En este ejemplo, la narración expuesta es la del niño como el sacrificio que hace la madre para su salvación. No la lleva a pensar en la calidad de vida que tiene, sino a soportar abnegadamente la suerte que le tocó vivir por la promesa de la gratificación. La entrevista de Luz se narra teniendo como ejes dos momentos significativos para su vida: el encarcelamiento de su esposo y la discapacidad de la hija desde su nacimiento. 
Caso 6. "Es que todas las noches le pido a Dios que me ayude a caminar, a hablar, pero no, no me escucha”.

Esta manera de aceptar la enfermedad por parte de los adultos, que constituye el pilar para valorar su experiencia, no siempre es compartida por los niños. En uno de los casos, los padres trataron de hablar con su hija sobre la discapacidad utilizando ese discurso, pero la niña se sintió profundamente irritada con ellos y con Dios debido a que no la escuchaba cuando le pedía caminar:

«Le digo: "Mi amor, mi padre Dios quiso que tú estuvieras así. Como todo el mundo somos diferentes, tú eres así, así Dios quiso mandarte". Y era cuando también se ponía triste: "Es que todas las noches le pido a Dios que me ayude a caminar, a hablar, pero no, no me escucha". Y le digo: "Hija, Dios está ocupado. Mira a tantos niños con cáncer, con tumores en su cerebro y que están a punto de morir. Él se preocupa por ellos. Porque a ti no te falta nada. Tú, gracias a Dios, puedes comer, puedes usar el baño... O sea, ¿qué te falta?" »".

Cabe destacar que Juanita (12 años, asiste a una escuela especial) no cumple con sus objetivos de rehabilitación, pues se niega a llevar a cabo sus terapias. Se muestra incómoda con su cuerpo y tiene continuos arranques de enojo. Para ella, la creencia en un Dios que quiere que ella tenga parálisis cerebral no constituye un elemento para valorar su vida como algo deseable, con metas, o lo suficientemente motivante para aspirar a estar mejor. El debate sobre un Dios que quiere que ella esté así se ha convertido en un tema de suma importancia, pues no puede conciliar a una divinidad así con el Dios benevolente de sus padres en virtud de que sus condiciones de vida y experiencias son muy distintas.

Sus padres, Lukas (44 años, primaria terminada) y Ramona (45 años, secundaria terminada), son creyentes devotos, y de esta manera han podido sobrellevar el problema de la discapacidad de Juanita. Tienen, además de ella, dos hijos: María (20 años, universidad) y Alberto (18 años, preparatoria). Los tres hermanos tienen muy buena relación entre sí.

\section{DISCUSIÓN}

Para Turner (1996), la relación entre la salud y la religión es obligada si se toma en cuenta que "la religión se ocupa de la pureza del alma y el cuerpo". Se considera entonces que en un estudio sobre el padecimiento físico son importantes las creencias religiosas como estrategia de afrontamiento de quienes lo padecen y de sus familias.

La mayoría de las familias participantes (once) que relacionaron la calidad de vida con sus creencias religiosas tenían en su seno a un menor con una discapacidad debida a la parálisis cerebral, es decir, a un dependiente total. Las siete familias restantes tenían problemas graves en su funcionamiento, con una evidente fractura en las relaciones interpersonales y sociales, asociadas con desventajas culturales. Aunque en este estudio es difícil afirmar que el tipo de discapacidad, las características sociodemográficas o el sufrimiento fortalecen o animan el apego a las creencias religiosas, hay estudios que encuentran una relación cuando la población tiene ciertas características. Por ejemplo, en un estudio realizado en México por Rivera y Montero (2007) con adultos mayores se encontró que el afrontamiento religioso de la soledad es una estrategia más común en las mujeres que en los hombres, pero conforme avanza la edad ambos sexos la utilizan. Dicha estrategia también es empleada más por personas con escaso nivel educativo, solteros o viudos y cristianos, en comparación con personas de mayor nivel educativo, casados o en unión libre y católicos, respectivamente.

Es un hecho que durante mucho tiempo se ignoró la relación entre la calidad de vida y las creencias religiosas. Los principales teóricos que exploran el bienestar de las personas con discapacidad y sus familias no consideran que las creencias religiosas sean un elemento a evaluar (Córdova, Verdugo y Gómez, 2009; Gómez y Verdugo, 2009; González y Jenaro, 2009; Sabeh, Verdugo y Prieto, 2009; Sainz, Verdugo y Delgado, 2009; Verdugo y Schalock, 2009). Es solo en los últimos años cuando han surgido estudiosos que encuentran una relación no solo indudable sino primordial (Murphy, Ciarrocchi, Piedmont, Cheston y Peirot, 2000; Quiceno y Vinaccia, 2011; Rodrí- 
guez, Fernández, Pérez y Noriega, 2011; Yoffe, 2009), autores para los cuales marca una diferencia importante en la percepción de bienestar, lo que coincide con los resultados del presente estudio.

Dichos hallazgos coinciden asimismo en mostrar una característica común: la necesidad de construir una narrativa que permita percibir la vida como algo tolerable o, en algunos casos, hasta próspera y con un bienestar evidente, lo que coincide con lo señalado por Kleinman (2008) sobre la relevancia de la narrativa en la comprensión profunda de la enfermedad, el cuidado (Kleinman, 1988), el sufrimiento y la calidad de vida (Kleinman y Kleinman, 1996). Las creencias religiosas en los casos de enfermedad o discapacidad se integran como una trama posible en la narración. De acuerdo con los discursos que se asuman, se construye la realidad de las experiencias. En el presente estudio se observa la manera en que las creencias religiosas se trenzan con el modo en que las personas perciben su calidad de vida en el continuo vaivén de la aceptación y la lucha: 1) en la mayoría de los casos representa para ellas un intento de adaptación individual y social (en algunas ocasiones afortunado, no así en otras); 2) aceptan con abnegación el sufrimiento por ser la voluntad de Dios, lo que les hace posible plantear su situación a través de valores culturalmente reconocidos y aceptados, un ejemplo de lo cual es la madre abnegada que sacrifica todo por sus hijos, y 3) se produce un cuestionamiento sobre su propia creencia en Dios.

Cuando las familias con un miembro discapacitado buscan una explicación, no es difícil encontrar respuestas del tipo "Dios así lo quiso". Esta idea es tratada por Swinburne (2011), quien explica que, en el contexto religioso, esta respuesta funciona debido a que contiene los elementos para afirmar la creencia: únicamente un mal puede ocurrir para lograr un bien mayor. Para las familias estudiadas en este trabajo, tal patrón se ejemplifica cuando asumen su condición como el modo en que la familia podrá mantenerse unida.

Por otro lado, se advierte el papel de la mujer en estos grupos familiares: abnegada, aislada y sin reconocer que ella también necesita bienestar (Mora, 2012). Esto mismo se explora en el trabajo de Landsman (2009) con madres de niños con discapacidad en Gran Bretaña: la mujer como cuidadora primaria suele cargar no únicamente con el trabajo que implica el cuidado del niño discapacitado, sino también con la culpa por esa discapacidad. En esta afirmación ocupa un lugar primordial la idea tan extendida de que es el cuerpo femenino en el que se desarrollan los niños el responsable, dejando de lado una gran cantidad de circunstancias por las que la discapacidad puede ocurrir.

Finalmente, respecto a cómo es que lo señalado aquí podría servir para el tratamiento de estos niños y de sus familias, se aprecia que la capacidad de las creencias para crear las tramas con las que se concibe la realidad es un campo que han considerado de interés diversas terapias; la narrativa con la que se "co-escribe" la realidad por los sujetos puede crear nuevos significados (Limón, 2005); esto es, que lo que se entiende por bienestar, calidad de vida y enfermedad son elementos de suma importancia en la atención de la salud. De aquí que las creencias deban ser integradas al tratamiento, ya sea desde una visión posmoderna, constructivista o sistémica, pues, como apunta Caballero (2014), las creencias guían la respuesta de los sujetos en el mundo.

En conclusión, el presente estudio propone en términos generales que el análisis de las creencias y las prácticas religiosas puede 1) contribuir a la evaluación de la calidad de vida de los niños con parálisis cerebral y de sus familias (incluso algunos de ellos expresan que la calidad de vida es “estar bien con Dios"); 2) alterar la dinámica de las familias, pues tales creencias les hacen posible soportar diversas situaciones por las promesas de su credo; 3) favorecer la reestructuración de la idea de enfermedad y permitir su resignificación, aunque no una mayor resiliencia ante el dolor; 4) ayudar en la resignación en el duelo ante la muerte; 5) modificar la conducta o la actitud ante la rehabilitación cuando no se corresponden con la idea del dios misericordioso que ayuda a la curación, y 6) dirigir la trama hacia una definición de la experiencia de la enfermedad.

En el presente estudio se reconoce que, para las madres entrevistadas, las creencias religiosas desempeñan un importante papel en su cotidianidad al ofrecerles "asideros de sentido"; son estas las que les hacen comprender y transitar en las vidas compartidas con sus familias, con ellas mis- 
mas y con su hijo enfermo. Por tanto, suelen ser benéficas y favorecer la formación de una experiencia más positiva y significativa. Sin embargo, cuando se interpretan a un grado tal que el propio bienestar se ve en un segundo término, vuelven vulnerables a los sujetos y tienen serias repercusiones en su salud física y mental que es necesario tratar mediante intervenciones que consideren, además de los elementos tradicionales, los culturales y religiosos. Otro aspecto por considerar es que las instituciones no pierdan de vista la importancia de dichos elementos y colaboren de manera eficiente en enviar los mensajes adecuados para que todos los miembros de la familia generen y colaboren en la gestión de mejores condiciones de bienestar para cada uno de ellos.

En ambos casos, es importante destacar que el entorno en el cual viven las familias tiene un peso importante en la manera en que se construye la vida tras la llegada de un niño con discapacidad. La idea del sufrimiento llevado hasta límites que no son sanos - como el de esperar que sean las madres sobre las que recaiga la mayor parte del cuidado y del sostén emocional primario de ella y su familia- puede tener y tiene efectos en la relación y calidad de vida de toda la familia.

El dolor, la enfermedad y el sufrimiento son procesos socioculturales que trascienden el mundo físico y enmarcan lo que el cuerpo hace a la vida de los sujetos que los padecen, y lo que la cultura, a través de las creencias religiosas, ofrece como un esquema perceptual para comprender su experiencia de vida.

Es necesario continuar el estudio de las creencias religiosas como una trama en la narrativa de vida de las personas que sufren enfermedades y sus familias, así como el modo en que aquellas les hace posible comprender su situación y vivirla desde el padecimiento, la culpa, el dolor y, en muchos casos, la precariedad de opciones disponibles para mejorar sus condiciones de bienestar y calidad de vida.

\section{REFERENCIAS}

Andéu, J. (2000). Las técnicas de análisis de contenido: una revisión actualizada. Fundación Centro Estudios Andaluces, 10(2), 1-3. Disponible en http://public.centrodeestudiosandaluces.es/pdfs/S200103.pdf.

Bateson, G. y Bateson, C. (2000). El temor de los ángeles. Epistemología de lo sagrado. Madrid: Gedisa.

Boyer, P. (2010). Y el hombre creó a los dioses. México: Taurus.

Caballero, E. (2014). Estructura mental de la patología. México: Psicoterapia de Premisas.

Córdova, L., Verdugo, M. y Gómez, J. (2009). Adaptación de la Escala de Calidad de Vida Familiar en Cali (Colombia). En M. Verdugo (Dir.): Como mejorar la calidad de vida de las personas con discapacidad. Instrumentos y herramientas de evaluación (pp. 273-290). Salamanca (España): Amarú.

Funes, M. (2007). Mito y ritual: terapeutas religiosos en el contexto del catolicismo en el área metropolitana (Argentina). Mitológicas, 22, 55-68.

Gómez, M. y Verdugo, M. (2009). La calidad de vida en la adolescencia: evaluación de jóvenes con discapacidad y sin ella. En M. Verdugo (Dir.): Como mejorar la calidad de vida de las personas con discapacidad. Instrumentos y herramientas de evaluación (pp. 77-102). Salamanca (España): Amarú.

González, F. y Jenaro, C. (2009). La calidad de vida en niños hospitalizados. En M. Verdugo (Dir.): Como mejorar la calidad de vida de las personas con discapacidad. Instrumentos y herramientas de evaluación (pp. 103-122). Salamanca (España): Amarú.

Kleinman, A. (1988). The illness narratives. Suffering, healing and human condition. New York: Basic Books.

Kleinman, A. (2008). Catastrophe and caregiving: the failure of medicine as an art. The Lancet, 371(9606), $22-23$.

Kleinman, A. y Kleinman, J. (1996). The appeal of experience; the dismay of images. Cultural appropriations of suffering in our times. Daedalus, 125(1), 1-23.

Landsman, G. (2009). Reconstructing motherhood and disability in the age of "perfect" babies. London: Routledge.

Limón, G. (2005). Terapias posmodernas. Aportaciones construccionistas. México: Pax México.

Lorente, M., Rosa, A., Montero, I., Martí, L., García, B., Calvo, E., Fierro, M., Martín, M., García, M., Martínez, M., Ortega, P., Gómez, M. y López, P. (1993). Instrucción y progreso escolar en niños con parálisis cerebral, de preescolar y ciclo inicial: un estudio de seguimiento. Madrid: Centro de Publicaciones del Ministerio de Educación y Ciencia.

Mora, C. (2012). Familias de pacientes con parálisis cerebral severa; sus indicadores de calidad de vida. Investigación y Ciencia, $55,33-41$. 
Murphy, P., Ciarrocchi, J., Piedmont, R., Cheston, S.H. y Peyrot, M. (2000). The relation of religious belief and practices: depression and hopelessness in persons with clinical depression. Journal of Consulting \& Clinical Psychology, 68(6), 1102-1106.

Parsons, T. (1996). The theory of human behavior in its individual and social aspects. The American Sociologist, $27(4), 13-23$.

Quiceno, J. y Vinaccia, S. (2011). Creencias-prácticas y afrontamiento espiritual-religioso y características sociodemográficas en enfermos crónicos. Psychology. Avances de la disciplina, 5(1), 25-36.

Ridge, D., Williams, I., Anderson, J. y Elford, J. (2008). Like a prayer: the role of spirituality and religion for people living with HIV in the UK. Sociology of Health \& Illness, 30(3), 413-428.

Rivera, A. y Montero, L. (2007). Medidas de afrontamiento religioso y espiritualidad en adultos mayores mexicanos. Salud Mental, 30(1), 39-47.

Rodríguez, M., Fernández, M., Pérez, M. y Noriega, R. (2011). Espiritualidad variable asociada a la resiliencia. Cuadernos Hispanoamericanos de Psicología, 11(2), 24-49.

Sabeh, E., Verdugo, M. y Prieto, G. (2009). Dimensiones e indicadores de calidad de vida en la infancia. En M. Verdugo (Dir.): Como mejorar la calidad de vida de las personas con discapacidad. Instrumentos y herramientas de evaluación (pp. 61-76). Salamanca (España): Amarú.

Sainz, F., Verdugo, M. y Delgado, J. (2009). Adaptación de la Escala de Calidad de Vida Familiar al contexto español. En M. Verdugo (Dir.): Como mejorar la calidad de vida de las personas con discapacidad. Instrumentos y herramientas de evaluación (pp. 251-270). Salamanca (España): Amarú.

Salazar, C. (2014). Antropología de las creencias. Religión, simbolismo, irracionalidad. Barcelona: Fragmenta.

Schalock, R. y Verdugo, M. (2009). Revisión actualizada del concepto de calidad de vida. En M. A. Verdugo (Dir.): Cómo mejorar la calidad de vida de las personas con discapacidad. Instrumentos y estrategias de valoración (pp. 29-41). Salamanca (España): Amarú.

Swinburne, R. (2011). La libertad y el mal. En J. Baggini y J. Stangroom (Comps.): Lo que piensan los filósofos (pp.113-120). Madrid: Paidós.

Turner, B. (1996). The body and society. London: SAGE.

Vallés M., S. (2009). Cuadernos metodológicos: entrevistas cualitativas. Madrid: Centro de Investigaciones Sociológicas.

Verdugo, M. y Schalock, R. (2009). Aspectos claves para medir la calidad de vida. En M. Verdugo (Dir.): Como mejorar la calidad de vida de las personas con discapacidad. Instrumentos y herramientas de evaluación (pp. 43-58). Salamanca (España): Amarú.

Verdugo, M., Jenaro C. y Schalock, R. (2009). Estudio transcultural de la calidad de vida. En M. A. Verdugo (Dir.): Cómo mejorar la calidad de vida de las personas con discapacidad. Instrumentos y estrategias de valoración (pp. 251-265). Salamanca (España): Amarú.

Yanez, B., Edmondson, D., Stanton, A., Park, C.L. Kwan, L. Ganz, P.A. y Blank, T.O. (2009). Facets of spirituality as predictors of adjustment to cancer: relative contributions of having faith and finding meaning. Journal of Consulting \& Clinical Psychology, 77, 730-41.

Yoffe, L. (2009). Efectos positivos de la religión y la espiritualidad en el afrontamiento de duelos. Psicodebate, 7, 193-206. Disponible en http://www.palermo.edu/cienciassociales/publicaciones/pdf/Psico7/7Psico\%2012.pdf. 